\title{
A Study of the Surface Plasmon Enhancement Using ARC on Thin Film Si Solar Cell Performance
}

\author{
Razan Nejm $^{1 *}$, Mousa Hussein ${ }^{1}$ and Ahmed Ayesh ${ }^{2}$ \\ ${ }^{1}$ Department of Electrical Engineering, United Arab Emirates University, Al Ain, United Arab Emirates \\ ${ }^{2}$ Department of Physics, United Arab Emirates University, Al Ain, United Arab Emirates
}

Received: November 30, 2013; Accepted: February 04, 2014; Published: February 09, 2014

"Corresponding author: Razan Nejm, Department of Electrical Engineering, United Arab Emirates University, Al Ain, United Arab Emirates, E-mail: r.radwan@uaeu.ac.ae

\begin{abstract}
Trapping light inside the solar cells can significantly boost their efficiency by minimizing the reflection losses at the front interface as well as the absorption losses in the solar cell by increasing the path length of incoming light. This study focuses on light trapping using single antireflection coating (ARC) and Ag nanoparticles (NPs). Theoretically $\mathrm{Si}_{3} \mathrm{~N}_{4}$ with quarter wave thickness allowed our Si ultrathin film device to absorb $70 \%$ of the incident light around the target wavelength before adding Ag NPs. However placing the Ag NPs on the ARC surface give the best results compared to the other positions, whereas decreasing the overall refractive index of the device improves its behavior.
\end{abstract}

Keywords: Plasmonic; Nanoparticle; Ultrathin film; Dielectric; Antireflection coating; Field enhancement

\section{Introduction}

Over the past ten years thin film solar cells have attracted the attention of optical researchers worldwide after reducing the material used in solar cell manufacturing $[1,2]$. This is due to the fact that $40 \%$ of a crystalline silicon solar module cost is the cost of the silicon material itself [3]. Naturally reducing the absorbing semiconductor thickness decreases the absorbing energy close to its electronic bandgap. This is also a major problem in Si thin film based devices, where Si have poor absorption near the bandgap as its absorption length is $>300 \mu \mathrm{m}[4,5]$.

Light trapping is the key solution for such energy-conversion efficiency, and it was achieved by different techniques such as surface texturing [6] and recently surface plasmon (SP) using metallic nanoparticles [3,7-11]. Although these two methods showed great improvement especially the later one, the main problem of solar cells is not solved which is light reflectance.

A significant part of the incoming light (up to 35\% approximately) is reflected by the solar cell surface due to the refractive index contrast between the ambient medium (air) and the solar cell silicon-based device [12,13]. Researchers found that covering the device by dielectric layer (antireflection coating) reduces the overall reflection of the incident light [1].
In result, this layer increases the amount of light entering the device and enhances the power conversion efficiency of solar cell [12]. Single layer antireflection coatings (ARC) designed with appropriate refractive index (n) and layer thickness (d) results in a significant decrease in the reflection coefficient $[14,15]$. One of the simplest and most efficient types of single ARC is called the quarter-wave transformer. Its great results make it a good and cheaper alternative for using metallic nanoparticles. However, using metallic nanoparticles with appropriate parameters might have a positive effect on the overall enhancement.

In our previous work [16], light scattering from silver (Ag) nanoparticles was investigated numerically by varying the adjustable parameters in order to determine some fundamental design principles. Light trapping using passivation layer (ARC) gave promised results. This paper studies the coupling of light into the Si substrate using ARC and Ag nanoparticles.

In this study we are focusing on ARC effect by changing its thickness, material and the nanoparticles position. The interparticle interaction between the nanoparticles is simulated using three Ag nanoparticles at different locations (on the surface of $\mathrm{ARC}$, on the surface of c-Si and on the middle of c-Si).

\section{Numerical Simulation}

Numerical simulations were performed using OptiFDTD simulation tool from Optiwave Company [17]. Three spherical silver (Ag) nanoparticles (NPs) with $30 \mathrm{~nm}$ diameter were modeled using Drude-Lorentz model. Crystalline silicon (c-Si) substrate of $100 \mathrm{~nm}$ in thickness and constant refractive index $(n=3.45)$ was used for all cases (semi-infinite and finite) models. All the results in this report are in term of electric field (Ey) which is limited by using 32-bit simulation type machine. Source is assumed normal incidence on the structure, propagating from air into substrate and 50nm above the device as shown in Figure 1.

The main goal of this study is to analyze the antireflection coating (ARC) on enhancing the c-Si efficiency in the presence of Ag nanoparticles. ARC is a thin layer that coats the thin film surface to minimize the reflection. 
Three different ARC materials are used in the analysis; these are $\mathrm{SiO} 2, \mathrm{Si}_{3} \mathrm{~N}_{4}$ and ITO. Drude-Lorentz model is used as well for modelling the ARC layers. APML boundary conditions are used throughout all the simulations. Three observation points were used to study the electric field intensity, on the surface of the device (including the passivation layer) (OP1), on the c-Si surface (OP2) and in the middle of the thin film (OP3). All the results are normalized to the incident electric field and calculated as a function of wavelength. The following section present results obtained based two scenarios Semi-infinite c-Si substrate and finite substrate.

\section{Results and Discussion}

\section{Semi-infinite c-Si substrate}

In order to get a better understanding of the ARC effect on the behavior of the model, $50 \mathrm{~nm}$ of ARC layer was added above the c-Si substrate and the results at OP1,OP2 and OP3, Figure 1 , recorded. To show the light trapping amount gained from adding the ARC, differential electric field between OP1 and OP3 is obtained as shown on Figure 2 for all three materials. $\mathrm{SiO}_{2}$ oxide layer gives the best enhancement with highest resonance, where $\mathrm{Si}_{3} \mathrm{~N}_{4}$ and ITO show almost similar results as there refractive indexes are close. These results are in agreement with [18].

Figure 2 highlights clearly redshift phenomena in $\mathrm{Si}_{3} \mathrm{~N}_{4}$ and ITO because of their higher refractive index. Increasing the ARC thickness increases the electric field trapped in the device as shown in Figure 3, where the thickness has been resulted in enhanced light trapping by approximately 10\%. A Redshift phenomenon is also clear in Figure 3 due to increasing the ARC thickness.

Changing passivation layer thickness shows direct proportion to electric field enhancement on the surface and inside the thin film. The thicker the dielectric layers the more intensity enhancement results, this is illustrated in Figure 4 taking $\mathrm{SiO}_{2}$ as an ARC material and changing its thickness $(t)$ from $20 \mathrm{~nm}$ to $90 \mathrm{~nm}$. One can notice from Figure 4 that the electric field trapped inside the device has increased as the thickness changed from 20 $90 \mathrm{~nm}$, moreover redshift is pronounce after 70nm thickness, this result agree with literature Akimov YK et al. [19].

This redshift phenomena allows manufacturer to tune the plasmon resonance of the solar cell as desired by changing the ARC material or thickness. In order to optimize ARC thickness and minimize incidence field reflectance from the device we

Figure 1: Device structure.

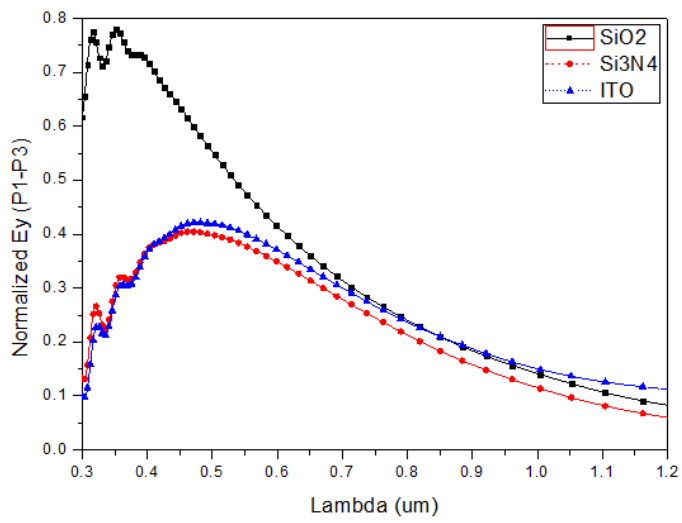

Figure 2: Effect of adding $50 \mathrm{~nm} \mathrm{ARC} \mathrm{on} \mathrm{semi} \mathrm{finite} \mathrm{c-Si.}$

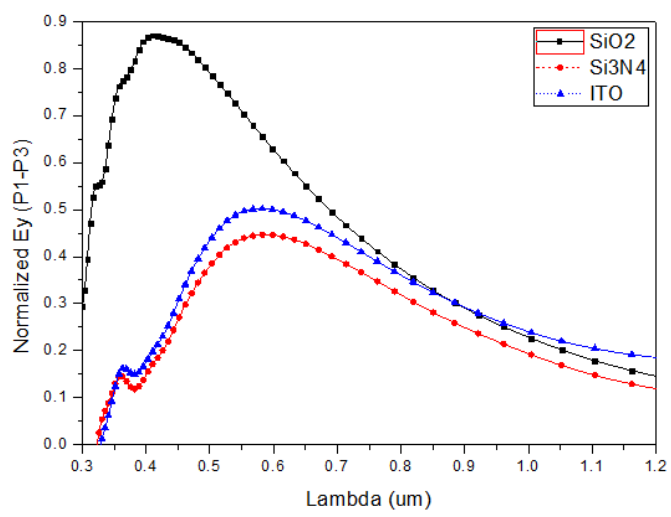

Figure 3: Effect of adding 70nm ARC on semi-finite $c$-Si.

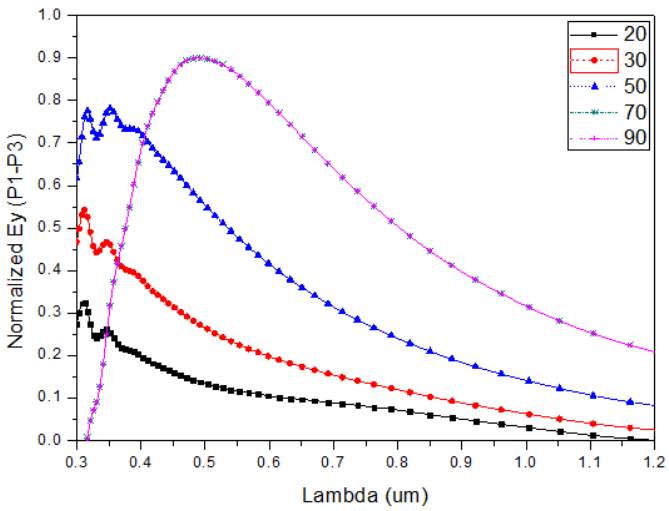

Figure 4: $\mathrm{SiO}_{2}$ effect as t changes.

propose using quarter wave transformer as a matching media between air and the c-Si substrate. This method depends on impedance matching method by forcing the electric length of the intermediate region (ARC) to be:

$$
t=\frac{\lambda}{4 n_{2}}
$$

Where $\lambda$ is the incident wave length and $n_{2}$ is the ARC real refractive index. 
Applying the quarter wave length matching theorem [20] $n_{2}$ can be found from:

$$
\begin{aligned}
& \frac{n_{1}-n_{2}}{n_{1}+n_{2}}=\frac{n_{2}-n_{3}}{n_{2}+n_{3}} \\
& n_{2}=\sqrt{n_{1} n_{3}}
\end{aligned}
$$

Where $n_{1}$ and $n_{3}$ are the refractive indices of the incident and the target regions respectively

In this study c-Si base solar cell with refractive index $\mathrm{n}_{3}=3.45$ is used as substrate, using (3) results in $\mathrm{n}_{2}=1.85 . \mathrm{Si}_{3} \mathrm{~N}_{4}$ and ITO both satisfy this condition according to [21] database. For $\lambda=550$ $\mathrm{nm}$, thickness of $\mathrm{Si}_{3} \mathrm{~N}_{4}$ and ITO $68 \mathrm{~nm}$ and $72 \mathrm{~nm}$ respectively from (3). Figure 5 shows the backscattering electric field from the three ARC materials for comparison. Although $\mathrm{SiO}_{2}$ doesn't satisfy the $\mathrm{n}_{2}$ condition [21], it is included in the analysis to prove the matching method.

As shown, $\mathrm{Si}_{3} \mathrm{~N}_{4}$ scattered the least amount of the incident electric field around the target wave length, whereas $\mathrm{SiO}_{2}$ gave the highest backscattering in spite of using the matching method. Figure 6 shows the absorbed electric field at the middle of the devise as comparison between $\mathrm{Si}_{3} \mathrm{~N}_{4}$ and ITO ARCs. $\mathrm{Si}_{3} \mathrm{~N}_{4}$ allows the device to absorb more electric field with wider resonance region. Less thickness and higher electric field absorption are two main reasons to make $\mathrm{Si}_{3} \mathrm{~N}_{4}$ preferable through this study.

All the results shown until now are before adding the Ag NPs. To show the advantage of adding ARC between the substrate and the NPs electric field was measured inside the c-Si at P3 for three different positions for the NPs as shown in Figure 7.

Ag NPs were placed on the top of the ARC $\left(\mathrm{Si}_{3} \mathrm{~N}_{4}+\mathrm{NPs}\right)$ partially inside the ARC $\left(\mathrm{Si}_{3} \mathrm{~N}_{4}+\mathrm{NPs}\right.$ in1) and on the surfaces of c-Si totally covered with ARC $\left(\mathrm{Si}_{3} \mathrm{~N}_{4}+\mathrm{NPs}\right.$ in 2$)$. As can be noticed in Figure 7 Adding NPs not always enhances the solar thin film absorption. Adding NPs on the ARC surface gave the best results inside the device, although not a big difference can be noticed after adding the NPs to the ARC layer.

From the backscattering electric field shown in Figure 8, it can be noticed that adding the NPs partially inside the ARC results in

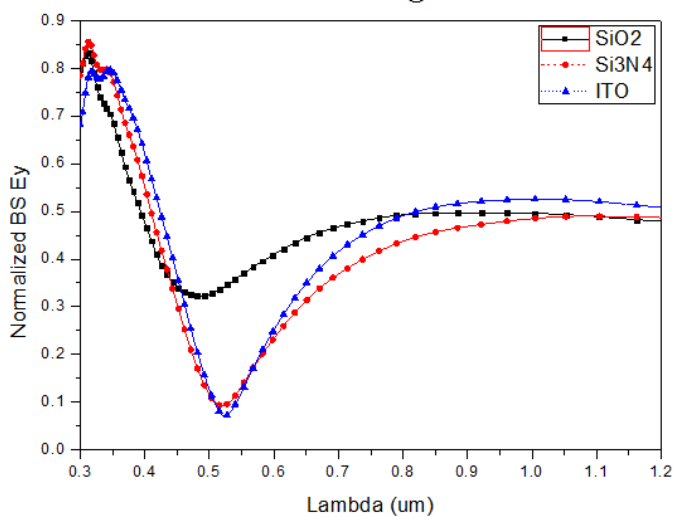

Figure 5: Ey backscattering from lambda $/ 4 \mathrm{n}_{2}$ ARC on semi finite c-Si.

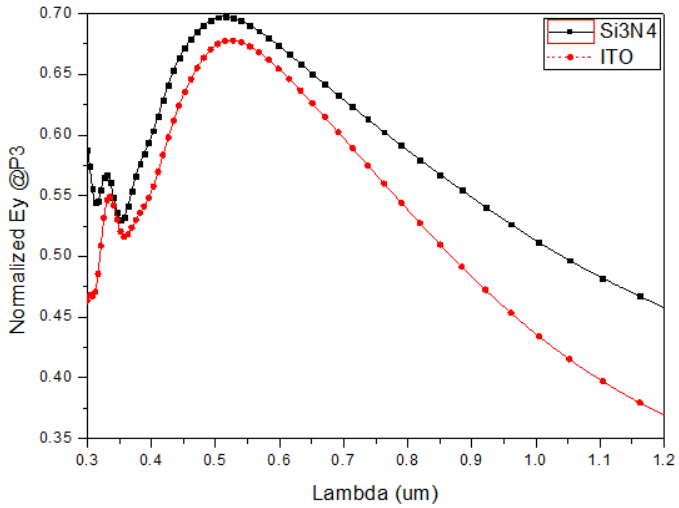

Figure 6: Ey@P3 for 68nm $\mathrm{Si}_{3} \mathrm{~N}_{4}$ and 72nm ITO ARCs.

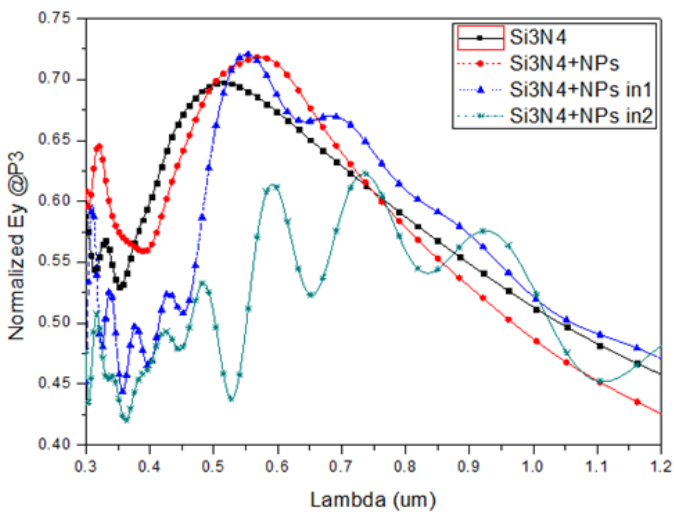

Figure 7: Ag NPs effect on Ey absorbing inside semi finite c-Si.

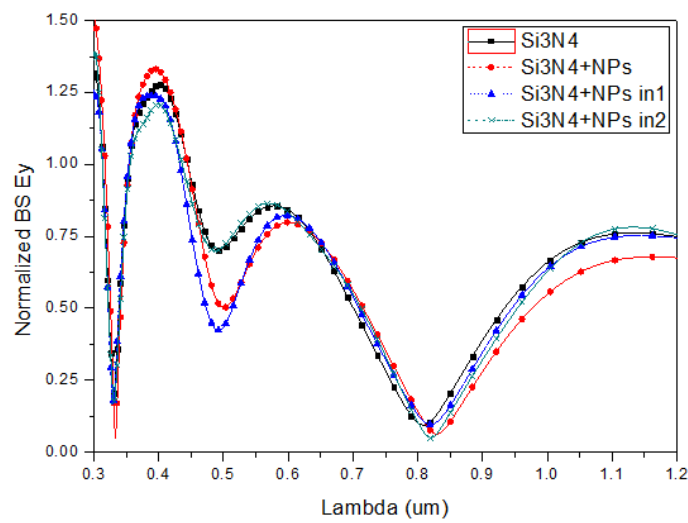

Figure 8: Ag NPs position effect on BS Ey.

less scattered field compared to the other cases although all the cases show very close results. This can be related to metal loss and back scattering which can be compensated by increasing the NPs diameter or number with appropriate spacing [16].

\section{Finite c-Si substrate}

Changing to finite substrate, the overall refractive index of the structure is reduced. All previous cases were repeated to study the effect of this reduction. 
The new obtained results showed higher electric field intensity and some results show almost double of the infinite case. Starting with the effect of adding $50 \mathrm{~nm}$ ARC to the solar ultrathin film $(100 \mathrm{~nm})$, Figure 9 shows almost double enhancement in electric field trapping for each ARC material.

Also increasing the ARC layer thickness in the finite case enhanced the absorbed electric field as shown in Figure 10. In both Figure $9 \& 10$ it can be noticed that two resonance peaks at least appeared compared to semi finite case Figure $2 \&$ Figure 3. Using the quarter wave transformer method for finding the best ARC refractive index in the finite case as in (3) didn't show good results compared to semi finite case. Figure 11 shows the backscattering electric field after adding matching ARCs.

Although in finite case we reached almost zero reflectance, we can notice $\mathrm{SiO}_{2}$ gave the best results this time compared to Figure 5 Also the minimum reflection value is not around the target wave length.

These results conclude that quarter wave transformer method is not compatible with finite case and correction parameters must be added.

Studying the effect of Ag NPs position on the absorbed electric field, NPs on the surface of the ARC shows clear enhancement

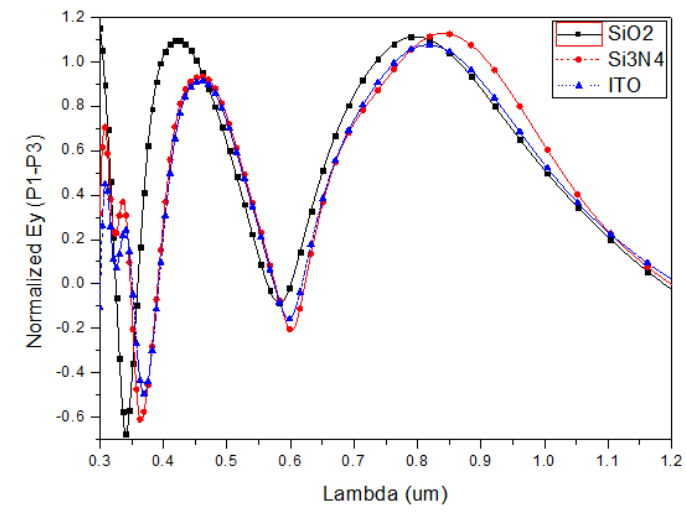

Figure 9: Effect of adding 50nm ARC on finite c-Si.

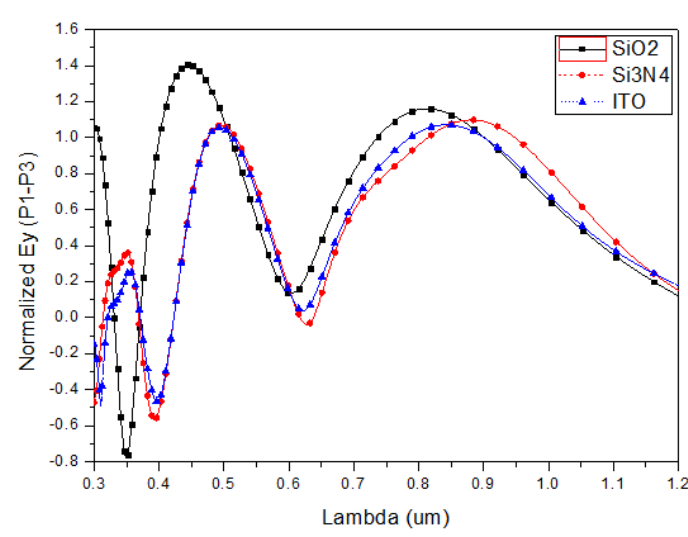

Figure 10: Effect of adding 70nm ARC on finite c-Si.

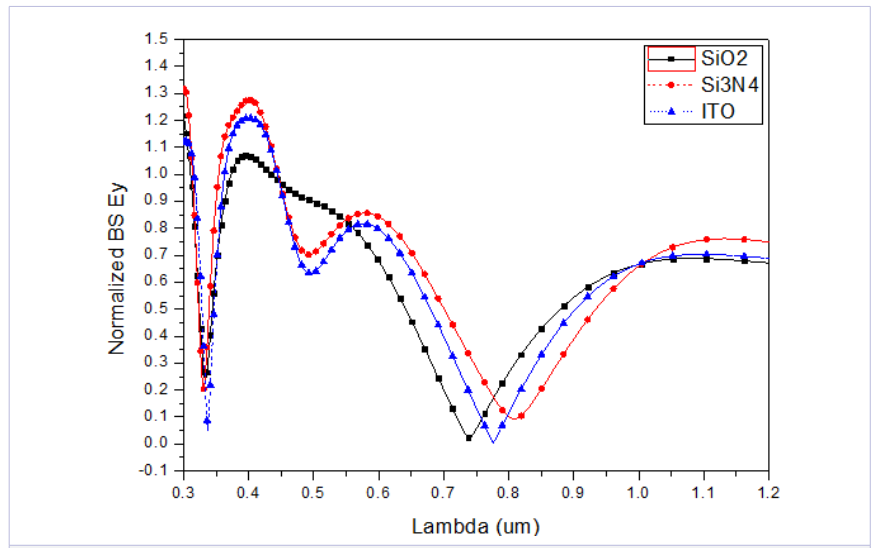

Figure 11: Ey backscattering from lambda $/ 4 n_{2}$ ARC on finite c-Si.

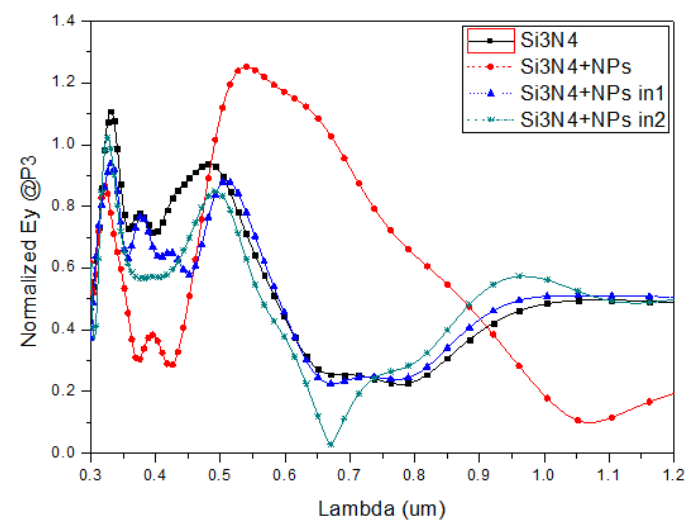

Figure 12: Ag NPs position effect on Ey absorbing inside finite c-Si.

compared to the other cases as in Figure 12 Compared to the semi-infinite case in Figure 7, all the positions showed better enhancement in the finite case.

\section{Conclusion}

This paper investigated the surface plasmon and light absorption enhancement of c-Si thin film by studying the ARC layer effect [22]. The main parameters in this study were the ARC thickness, material and NPs position. Finite and semi finite c-Si substrates are the structures for the carried simulation.

It was found that finite structure is preferable to improve the weak resonance region and decreasing the overall refractive index in general can improve the device behavior and this agrees with [16] results. Dielectric refractive index can be changed to tune the resonance location. Different materials can be used as ARC and get almost close results according to quarter wave transformer method but two parameters will be crucial, the target wave length and the ARC thickness. Placing the NPs on the ARC is preferable in both cases where electric field absorption enhanced inside the thin film.

\section{Acknowledgment}

The authors wish to acknowledge the support provided by the United Arab Emirates University. 


\section{References}

1. Green MA, Pillai S (2012) Harnessing plasmonics for solar cells. Nat Photon 6(3): 130-132.

2. Singh YP, Kumar A, Jain A, Kapoor A (2013) Enhancement in Optical Absorption of Plasmonic Solar Cells. The Open Renewable Energy Journal. 6(1): 1-6.

3. Catchpole KR, Polman A (2008) Plasmonic solar cells. Opt Express 16(26), 21793-21800.

4. Spinelli P, Ferry VE, van de Groep J, van Lare M, Verschuuren MA, et al. (2012) Plasmonic light trapping in thin-film Si solar cells. Journal of Optics 14(2)

5. Mokkapati S, Beck FJ, Polman A, Catchpole KR (2009) Designing periodic arrays of metal nanoparticles for light-trapping applications in solar cells. Applied Physics Letters 95(5).

6. Gangopadhyay U, Dhungel SK, Basu PK, Dutta SK, Saha H, et al. (2007) Comparative study of different approaches of multicrystalline silicon texturing for solar cell fabrication. Solar Energy Materials and Solar Cells 91(4): 285-289.

7. Spinelli P, Hebbink M, de Waele R, Black L, Lenzmann F, et al. (2011) optical impedance matching using coupled plasmonic nanoparticle arrays. Nano Lett 11(4): 1760-1765.

8. Ferry VE, Munday JN, Atwater HA (2010) Design considerations for plasmonic photovoltaics. Advanced Materials 22(43): 4794-4808.

9. Pillai S, Green MA (2010) Plasmonics for photovoltaic applications. Solar Energy Materials and Solar Cells 94(9): 1481-1486.

10. Pillai S, Catchpole KR, Trupke T, Green MA (2007) Surface plasmon enhanced silicon solar cells. J Appl Phys 101(9): 093105-093108.

11. Schaadt DM, Feng B, Yu ET (2005) Enhanced semiconductor optical absorption via surface plasmon excitation in metal nanoparticles. Applied Physics Letters 86(6): 063106.

12. Yan X, Poxson DJ, Cho J, Welser RE, Sood AK, et al. (2013) Enhanced omnidirectional photovoltaic performance of solar cells using multiple-discrete-layer tailored- and low-refractive index antireflection coatings. Advanced Functional Materials 23(5): 583-590.

13. Aroutiounian VM, Martirosyan KH, Soukiassian P (2006) Almost zero reflectance of a silicon oxynitride/porous silicon double layer antireflection coating for silicon photovoltaic cells. Journal of Physics D: Applied Physics 39(8).

14. Richards BS (2004) Comparison of $\mathrm{TiO}_{2}$ and other dielectric coatings for buried-contact solar cells: a review. Progress in Photovoltaics: Research and Applications 12(4): 253-281.

15. Aroutiounian VM, Martirosyan KH, Soukiassian P (2004) Low reflectance of diamond-like carbon/porous silicon double layer antireflection coating for silicon solar cells. Journal of Physics D: Applied Physics 37(19).

16. Nejm R, Hussein MI, Ayesh A (2013) Design consideration for plasmonic solar cells based on Ag nanoparticles. 4th International Conference on Metamaterials, Photonic Crystals and Plasmonics (META13). Sharjah, United Arab Emirates.

17.http://optiwave.com

18. Beck FJ, Polman A, Catchpole KR (2009) Tunable light trapping for solar cells using localized surface plasmons. Journal of Applied Physics 105(11): 114310-114317.

19. Akimov Y, Ostrikov K, Li EP (2009) Surface plasmon enhancement of optical absorption in thin-film silicon solar cells. Plasmonics 4(2): 107-113.

20.Sadiku MNO (2010) Elements of electromagnetic. Oxford University Press Inc., New York, USA.

21. http://refractiveindex.info/

22. Marrocco V, Grande M, Vincenti MA, Calo G, Petruzzelli V, et al. (2010) Efficient plasmonic nanostructures for thin film solar cells. Photonics for Solar Energy Systems III 7725, doi:10.1117/12.854495. 